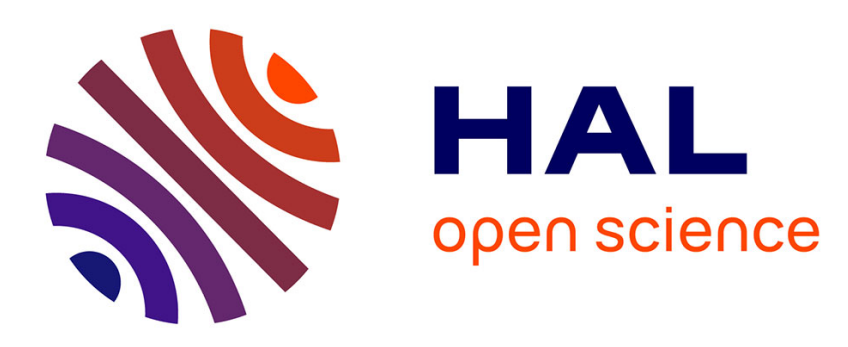

\title{
Terahertz time domain spectroscopy of phonon-depopulation based quantum cascade lasers
}

Nathan Jukam, Sukhdeep Dhillon, Dimitri Oustinov, Julien Madéo, Jérôme

Tignon, Raffaele Colombelli, Paul Dean, Mohammed Salih, Suraj P. Khanna, Edmund H. Linfield, et al.

\section{To cite this version:}

Nathan Jukam, Sukhdeep Dhillon, Dimitri Oustinov, Julien Madéo, Jérôme Tignon, et al.. Terahertz time domain spectroscopy of phonon-depopulation based quantum cascade lasers. Applied Physics Letters, 2009, 94, pp.251108. hal-00401507

\section{HAL Id: hal-00401507 https://hal.science/hal-00401507}

Submitted on 3 Jul 2009

HAL is a multi-disciplinary open access archive for the deposit and dissemination of scientific research documents, whether they are published or not. The documents may come from teaching and research institutions in France or abroad, or from public or private research centers.
L'archive ouverte pluridisciplinaire HAL, est destinée au dépôt et à la diffusion de documents scientifiques de niveau recherche, publiés ou non, émanant des établissements d'enseignement et de recherche français ou étrangers, des laboratoires publics ou privés. 
Terahertz time domain spectroscopy of phonon-depopulation based quantum cascade lasers

\author{
N. Jukam, S. S. Dhillon, D. Oustinov, J. Madéo and J. Tignon \\ Laboratoire Pierre Aigrain, Ecole Normale Supérieure, UMR8551 CNRS, 75231 Paris Cedex \\ 05, France. \\ R. Colombelli
}

Institut d'Electronique Fondamentale, Université Paris Sud, UMR8622 CNRS, 91405 Orsay, France

P. Dean, M. Salih, S. P. Khanna, E. H. Linfield and A. G. Davies

School of Electronic and Electrical Engineering, University of Leeds, Leeds LS2 9JT, UK 


\begin{abstract}
The gain and loss in a terahertz quantum cascade laser based on a longitudinal optical phonon depopulation scheme is studied using terahertz time domain spectroscopy. At laser threshold the gain is found to be clamped at $25 \mathrm{~cm}^{-1}$ for a $1 \mathrm{~mm}$ long device and the full width at half maximum of the spectral gain during laser action is $0.6 \mathrm{THz}$. At low biases, before laser action, significant absorption features are observed for frequencies lower and higher than the laser emission frequency. We assign their onset to the lower laser level being populated from a parasitic electronic channel. The absorption transitions are identified from bandstructure simulations and from the oscillator strengths of the subband transitions as a function of the applied field.
\end{abstract}


Terahertz (THz) quantum cascade lasers (QCLs) based on longitudinal-optical (LO) phonon depopulation of the lower laser level ${ }^{1}$ are currently attracting considerable interest, owing to their higher output powers ${ }^{2}$ and operating temperatures ${ }^{3}$ compared to bound-tocontinuum designs. ${ }^{4}$ Recently time domain spectroscopy (TDS) has been used to study the spectral gain of THz and mid-infrared QCLs. ${ }^{5,6}$ Broadband THz probe pulses are coupled into the laser cavity and the detection of the transmitted pulses is used to determine the spectral gain. Owing to the coherent nature of the detection only the transmitted probe pulses are measured, which enables measurements of the spectral gain both below and above laser threshold. ${ }^{7,8}$ Previous TDS studies of THz QCLs have focused on bound-to-continuum designs while no studies on devices based on the LO phonon depopulation scheme have been reported to date.

In this letter we investigate the gain of a $3.1 \mathrm{THz}$ LO phonon QCL using THz TDS. The sample is based on the three well structure by Luo et $\mathrm{al}^{9}$ which has subsequently shown operation up to $178 \mathrm{~K}$ in a double metal waveguide. ${ }^{10}$ In addition, we identify various absorption features at low fields, before the bandstructure aligns for laser action. These absorptions correspond to the pre-alignment of the structure and the population of the lower laser level through an electronic channel.

The QCL consists of 226 periods of a series of $\mathrm{Al}_{0.15} \mathrm{Ga}_{0.85} \mathrm{As}$ barriers and GaAs quantum wells with thicknesses 4.2/7.4/2/9.6/4.8/16.1 nm (AlGaAs barriers in bold). Compared to the QCL in reference 10, this structure has a much thicker lower doped layer (700nm GaAs, doped at $2 \times 10^{18} \mathrm{~cm}^{-3}$ ) to support waveguiding in a single plasmon configuration, this waveguide geometry being used to enable coupling of the $\mathrm{THz}$ pulses into the laser cavity. The ridge width of the device was $140 \mu \mathrm{m}$ and the cavity length was $1 \mathrm{~mm}$. 
The device showed laser action up to a heat sink temperature of $85 \mathrm{~K}$ at a $2.5 \%$ duty cycle and $70 \mathrm{~K}$ at $10 \%$ duty cycle. The laser emission was centered at $\sim 3.1 \mathrm{THz}$ (see inset, Fig. 1(c)).

Broadband $\mathrm{THz}$ probe pulses were generated using an inter-digitated photoconductive antenna ${ }^{11}$ excited with a Ti:Sapphire femtosecond laser operating at $76 \mathrm{MHz}$. The THz probe pulses were coupled into an end facet of the laser, with the transmitted pulses collected from the opposite facet and detected by a $300 \mu \mathrm{m}$ thick GaP crystal using electro-optic sampling. ${ }^{12}$ The spectral gain was determined using a transmitted reference pulse with the QCL under zero bias. Details of this technique can be found in reference 7.

Fig. 1(a) shows the voltage-current (V-I) and light-current (L-I) characteristics of the laser at $10 \mathrm{~K}$, the latter measured with a standard pyroelectric detector. The laser threshold occurs at current density of $1.07 \mathrm{kA} / \mathrm{cm}^{2}$ and laser action ceases at $1.37 \mathrm{kA} / \mathrm{cm}^{2}$. Fig. 1(b) shows the difference transmitted electric fields (i.e. that measured relative to the modulation frequency of the QCL) ${ }^{7}$ of the THz pulses for various current densities. For current densities greater than $792 \mathrm{~A} / \mathrm{cm}^{2}$ (when the bandstructure starts to align up to the design field) oscillations of the field are observed owing to the amplification of the input pulse and last for approximately $3 \mathrm{ps}$ when the device is above threshold. The short time period of the oscillations, compared to bound-to-continuum designs, ${ }^{7,8}$ are attributed to both the small cavity length ${ }^{13}$ and the broad gain spectra. The latter can be observed in Fig. 1(c) which shows the gain spectra for three different currents with maximum gain at $3.11 \mathrm{THz}$, in accordance with the design frequency $(3.1 \mathrm{THz})$. The linewidth of the gain spectrum is 0.6 $\mathrm{THz}$ at laser threshold, compared to $0.32 \mathrm{THz}$ for a $2.9 \mathrm{THz}$ bound-to-continuum design. ${ }^{5}$ The reason for this large linewidth is possibly the larger number of periods (226 compared to 90), which leads to inhomogeneous broadening of the gain profile. This has been indirectly confirmed by measurements of the laser spectra of samples with a lower number of periods ${ }^{14}$ - these exhibit, for the same cavity length, a reduced number of Fabry-Perot modes and hence 
indicates a smaller gain linewidth. Other effects that could play a role include impurity scattering, ${ }^{15}$ the injector/laser state coupling ${ }^{8}$ and the higher electron temperature in LO phonon depopulation designs leading to a larger in-plane momentum. ${ }^{16} \mathrm{~A}$ very small narrowing of the spectral gain of approximately $80 \mathrm{GHz}$ is observed owing to the misalignment of the structure as the applied field is increased. ${ }^{8}$ The amplitude of the gain at 3.11 $\mathrm{THz}$ (i.e. at the laser emission frequency) as a function of applied current is also plotted in Fig. 1(a). During laser action the gain is clamped at a value of $\sim 25 \mathrm{~cm}^{-1}$. This value is in good agreement with a simple estimate of the total optical losses $\left(\alpha_{\text {Total }}=\alpha_{\mathrm{m}}+\alpha_{\mathrm{g}}=10.2 \mathrm{~cm}^{-1}+9.8 \mathrm{~cm}^{-}\right.$ ${ }^{1}=20 \mathrm{~cm}^{-1}$ where $\alpha_{\mathrm{m}}$ and $\alpha_{\mathrm{g}}$ are the mirror and waveguide losses respectively).

Compared to previous work on bound-to-continuum samples, ${ }^{7,8}$ transmitted $\mathrm{THz}$ electric fields at lower current densities (Fig. 1b) show several unusual features. As the current density is decreased from laser action $\left(1.17 \mathrm{kA} / \mathrm{cm}^{2}\right)$ to just below threshold $\left(0.92 \mathrm{kA} / \mathrm{cm}^{2}\right)$ and then to $0.79 \mathrm{kA} / \mathrm{cm}^{2}$, the amplitude and extent of the field oscillations decrease as expected. This corresponds to a decrease in the gain, and the transmission of the input pulse without amplification. However, below $0.79 \mathrm{kA} / \mathrm{cm}^{2}$, the transmitted amplitude unexpectedly increases again and long lasting oscillations appear after 10ps corresponding to a narrow linewidth spectral feature (with high frequency components at $\sim 4 \mathrm{THz}-$ see Fig. 3). The first few oscillations in the time trace correspond to a broad spectral response. The vertical line at $9.5 \mathrm{ps}$ in Fig. $1 \mathrm{~b}$ shows that the relative phase of the fields is nearly constant for current densities larger than $0.79 \mathrm{kA} / \mathrm{cm}^{2}$. Below $0.79 \mathrm{kA} / \mathrm{cm}^{2}$, however, the phase shifts significantly (by almost $180^{\circ}$ ) indicating a change from gain to strong absorption in the spectra. $^{5}$

The spectral gain below $0.80 \mathrm{kA} / \mathrm{cm}^{2}$ is shown in Fig. 2(a) and shows several features that are not present at higher current densities. For low frequencies, below the laser frequency of $3.1 \mathrm{THz}$, a broad absorption is present. Its peak red-shifts towards lower frequencies with 
increasing current densities, indicating a diagonal transition between adjacent wells. The narrow absorption at high frequencies, however, remains roughly constant in frequency at 4 4.2 THz, suggesting a vertical intersubband transition. Figs. 2(b) and 2(c) show the peak gain value and the corresponding frequency, respectively, of the low and high frequency absorptions as a function of current.

Simulations of the QCL's conduction band profile and the square modulus of the wavefunctions of the structure are shown in Fig. 3 for various applied fields (a-c). Corresponding schematic representations of the subband levels are also shown (d-f). The laser transition occurs within the two narrow wells separated by a thin barrier; the extraction and injection of electrons from one period to the next takes place in the wide well. The energy separation between the first two states in the wide well (labeled 4' and 2) is approximately the LO phonon energy to provide rapid scattering of electrons from the lower laser level to the first state of the wide well (4'). At the laser design bias of $12.5 \mathrm{kV} / \mathrm{cm}$ (Figs. 3(a) and (d)) the upper laser state (3) is resonant with the 'injector' state (4), and the lower state of the two narrow wells (1) is resonant with the second state ('extractor') of the wide well (2). As the applied field is reduced the injector (extractor) state decreases (increases) in energy with respect to the states in the two narrow wells. For intermediate fields $(9 \mathrm{kV} / \mathrm{cm}-$ Figs. $3(\mathrm{~b})$ and (e)) the injector (4) and extractor (2) levels are resonant although the overlap between the two states is small owing to their large spatial separation. For even lower fields $(7 \mathrm{kV} / \mathrm{cm}-$ Figs. 3(c) and (f)) the upper laser state (3) and extractor state (2), along with the lower level of the narrow wells (1) and injector (4) states, become resonant in energy.

At low biases, various absorption transitions between states $|1>| 2>,, \mid 3>$ and $\mid 4>$ are possible. A contour plot of all possible transitions is shown in Fig. 4 where the frequency separation and oscillator strength are plotted as a function of applied electric field. Encircled in Fig. 4 are the transitions that correspond to the observed absorption features at low current 
densities $\left(<0.8 \mathrm{kA} / \mathrm{cm}^{2}\right)$. The absorption observed at $4.2 \mathrm{THz}$ in Fig. 2 at low biases can be identified as the transition between states $\mid 1>$ and $\mid 3>$ as it does not shift significantly between $7 \mathrm{kV} / \mathrm{cm}$ and $11 \mathrm{kV} / \mathrm{cm}$, its oscillator strength disappears as the structure aligns for laser action and its FWHM $(\sim 0.27 \mathrm{THz})$ corresponds to a transition involving only two distinct states. The absorption peak at $\sim 4.6 \mathrm{THz}$ is an artifact of the measurement technique as the signal-to-noise ratio of the antenna signal is small for frequencies greater than $4.5 \mathrm{THz}$. The broad absorption at lower frequencies $(\sim 2 \mathrm{THz})$ in Fig. 2 is identified as the transition between the lower (1) and the extractor (2) level. The simulations in Fig. 4 show that this transition shifts to lower frequencies with increasing bias, which is in agreement with the observed behavior in Fig. 2. (As state 2 is resonantly depopulated through LO phonon emission to state 4' the electron population is expected to be very small in this state). ${ }^{17}$ The broadness of the absorption originates from its spatially diagonal character and also from the presence of nearby transitions (1-4, 2-4 and 2-3) whose dipoles contribute to the absorption spectrum for fields less than $10 \mathrm{kV} / \mathrm{cm}$. (A peak that corresponds to gain is also observed at 3.5 THz in Fig. 2. In the bandstructure simulations in Fig. 4, however, no such peak can be identified. This is possibly a result of domain formation and hence a non-uniform field across the active region). Note that the absorptions identified are a result of transitions from state 1 , meaning that most of the electrons are in this state at low applied fields. This corresponds to an electron leakage channel as these electrons are not injected into the upper laser state (level 3). Further improvements in the QCL design can therefore be achieved by suppressing this electronic channel by better injection schemes, allowing laser action to be obtained at lower threshold currents. This could in turn lead to a larger current dynamic range and a lower electron temperature, and achievement of higher operating temperatures.

In conclusion we have studied the gain and losses in LO phonon depopulation based THz QCLs. The clamped gain was found to be $25 \mathrm{~cm}^{-1}$ and agrees with a simple estimate of 
the total losses. Absorption features were also identified at low applied fields, before alignment of the energy levels for laser action, and correlated with bandstructure calculations. The absorptions are a result of a leakage current channel which leads to significant electronic population of the lower laser state before the structure is correctly aligned for laser action. The presented results suggest that the information provided by THz TDS can be valuable to elucidate parasitic transport mechanisms in QCLs.

This work was financially supported by the DGA, CNano, ANR, EPSRC and the EC NOTES programme. The LPA-ENS is a "Unité Mixte de Recherche Associée au CNRS UMR8551 et aux Universités Paris 6 et 7". 


\section{Figure Captions}

Figure 1: (a) Voltage, output power and gain at 3.11THz of the QCL as a function of current density at $10 \mathrm{~K}$. (b) Transmitted difference electric fields for various current densities at $10 \mathrm{~K}$. The applied duty cycle to the QCL is $15 \%$. The blue and green shaded regions correspond to low current densities before laser alignment and gain at the laser frequency when the bandstructure aligns, respectively. At low current densities $\left(65 \mathrm{~A} / \mathrm{cm}^{2}\right.$ to $\left.672 \mathrm{~A} / \mathrm{cm}^{2}\right)$ long lasting field oscillations are observed. At $792 \mathrm{~A} / \mathrm{cm}^{2}$, these oscillations disappear and a change in phase is observed for the first few oscillations. As the current density is increased further, field oscillations are observed from amplification at the laser frequency. Curves are offset for clarity. (c) Spectral gain at $10 \mathrm{~K}$ for various current densities $\left(1110 \mathrm{~A} / \mathrm{cm}^{2}\right.$ (red), $1258 \mathrm{~A} / \mathrm{cm}^{2}$ (green) and $1344 \mathrm{~A} / \mathrm{cm}^{2}$ (blue)) during laser action. Inset shows laser spectrum of equivalent device at a current density of $1176 \mathrm{~A} / \mathrm{cm}^{2}$ with laser emission at $3.11 \mathrm{THz}$.

Figure 2: (a) Spectral gain at low current densities showing a sharp absorption at $4.2 \mathrm{THz}$ and a broad absorption that shifts from $2.5 \mathrm{THz}$ to $1.75 \mathrm{THz}$ as the current density is increased. (Spectra offset for clarity). All features disappear as the current density is increased and the subbands align for laser action at $3.11 \mathrm{THz}$. The current density range of $137 \mathrm{~A} / \mathrm{cm}^{2}$ to $792 \mathrm{~A} / \mathrm{cm}^{2}$ corresponds approximately to applied fields of $5 \mathrm{kV} / \mathrm{cm}$ to $10 \mathrm{kV} / \mathrm{cm}$. The applied fields are obtained from the V-I characteristics after subtracting an estimate for the contact resistance contribution. b) Gain and (c) frequency position as a function of applied current for the low and high frequency absorptions.

Figure 3: Conduction band profile and modulus squared of the wavefunctions at applied fields of (a) $12.5 \mathrm{kV} / \mathrm{cm}$ where transitions $\mid 4>$ and $\mid 3>$ are resonant; (b) $9 \mathrm{kV} / \mathrm{cm}$; and (c) 
$7 \mathrm{kV} / \mathrm{cm}$. (d), (e) and (f) give corresponding schematic diagrams of the key energy states. (Bandstructure calculations performed using design dimensions).

Figure 4: Contour plot of intersubband transitions showing the oscillator strength (color scale on right) as a function of applied field and the frequency separation of the levels. The vertical line at $12.5 \mathrm{kV} / \mathrm{cm}$ corresponds to the laser alignment field where an anticrossing of the transitions 4-2 and 3-2 occurs, with each transition contributing equally to the spectral gain. At lower fields, the predominant absorptions observed in the experimental data correspond to transitions $|1>-| 3>$ and $|1>-| 2>$. 


\section{References}

1. B. S. Williams, H. Callebaut, S. Kumar, Q. Hu, and J. L. Reno, Appl. Phys. Lett. 82, 1015 (2003).

2. B. S. Williams, S. Kumar, Q. Hu, and J. L. Reno, Electronic Letters 42, 89 (2006).

3. B. S. Williams, Nature Photonics 1, 517 (2007).

4. S. Barbieri, J. Alton, H. E. Beere, J. Fowler, E. H. Linfield, and D. A. Ritchie, Appl. Phys. Lett. 85, 1674 (2004)

5. J. Kroll, J. Darmo, S. S. Dhillon, X. Marcadet, M. Calligaro, C. Sirtori, and K. Unterrainer, Nature 449, 698 (2007).

6. W. Parz, T. Muller, J. Darmo, K. Unterrainer, M Austerer, G. Strasser, L. R. Wilson, J. W. Cockburn, A. B. Krysa, and J. S. Roberts, Appl. Phys. Lett. 93, 091105 (2008).

7. N. Jukam, S. Dhillon, Z.-Y. Zhao, G. Duerr, J. Armijo, N. Sirmons, S. Hameau, S. Barbieri, P. Filloux, C. Sirtori, X. Marcadet, and J. Tignon, IEEE. J. Sel. Topics in Quantum Electronics 14, 436 (2008).

8. N. Jukam, S. S. Dhillon, D. Oustinov, Z.-Y. Zhao, S. Hameau, J. Tignon, S. Barbieri, A. Vasanelli, P. Filloux, C. Sirtori, and X. Marcadet, Appl. Phys Lett. 93, 101115 (2008).

9. H. Luo, S. R. Laframboise, Z. R. Wasilewsik, G. C. Aers, H. C. Liu, and J. C. Cao, Appl. Phys. Lett. 90, 041112 (2007).

10. M. A. Belkin, J. A. Fan, S. Hormoz, F. Capasso, S. P. Khanna, M. Lachab, A. G. Davies, E. H. Linfield, Optics Express 16, 3242 (2008).

11. A. Dreyhaupt, S. Winnerl, T. Dekorsry, and H. Helm, Appl. Phys. Lett 86, 121114 (2005).

12. Q. Wu and X. C. Zhang, Appl. Phys. Lett. 70, 1784 (1997). 
13. If the gain is clamped at frequency $v_{0}$ (such that the gain $\gamma\left(v_{0}\right)$ equals the total losses $\left.\alpha_{\text {Total }}\right)$, then the probe signal at frequency $v$ will be proportional to $\exp \left[\left(\gamma(v)-\gamma\left(v_{0}\right)\right)\right.$ L]. For $\gamma(v) \neq \gamma\left(v_{0}\right)$ the probe signal will increase (decrease) at $\gamma(v)$ as the cavity length decreases (increases) leading to broader (narrower) linewidth. Thus the raw spectral signal (i.e. the Fourier transform of the difference electric field) of the probe pulses will broaden and the field oscillations will become shorter with decreasing cavity length.

14. Y. Chassagneux, J. Palomo, R. Colombelli, S. Barbieri, S. Dhillon, C. Sirtori, H. E. Beere, J. Alton, D. A. Ritchie, El. Lett., 43, 285 (2007).

15. R. Nelander and A Wacker, Appl. Phys. Lett. 92, 081102 (2008).

16. M S. Vitiello, G. Scamarcio, V. Spagnolo, B. S. Williams, S. Kumar, Q. Hu, and J. L. Reno, Appl. Phys. Lett. 86, 11115 (2005).

17. As state 2 and 4' are mostly in the wide well of the structure, the energy separation does not vary significantly with applied field and is always close to the LO phonon energy. This will lead to carriers in state 2 being resonantly depopulated for the all applied fields. 


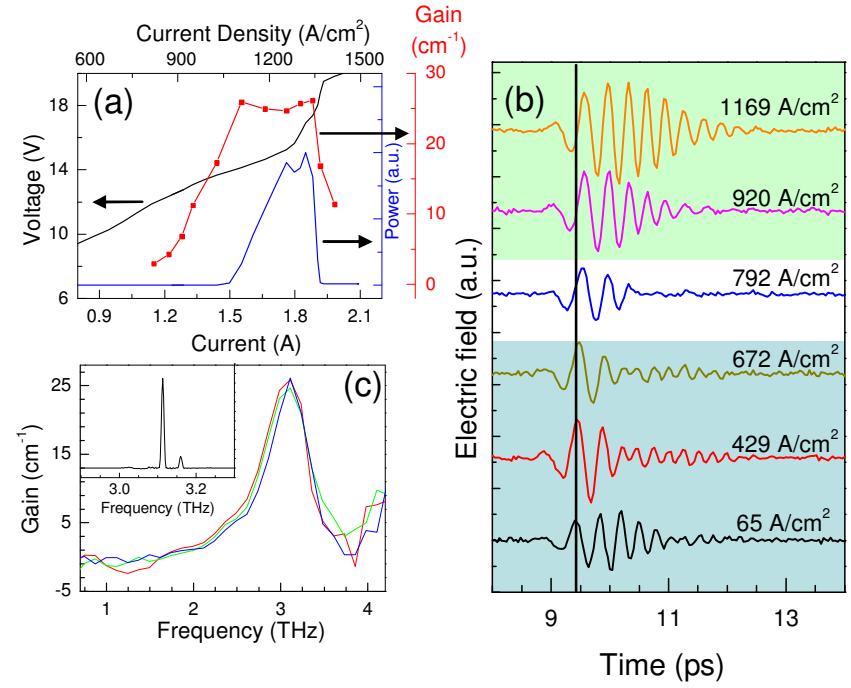




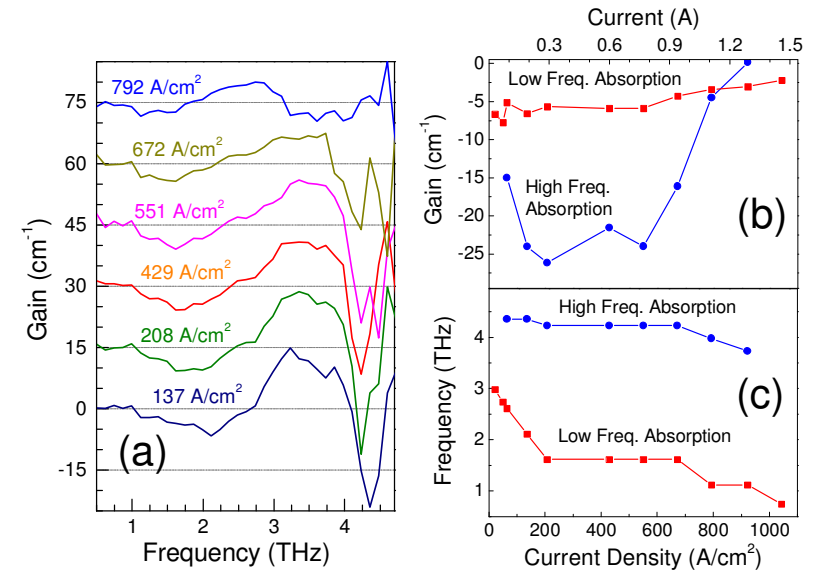



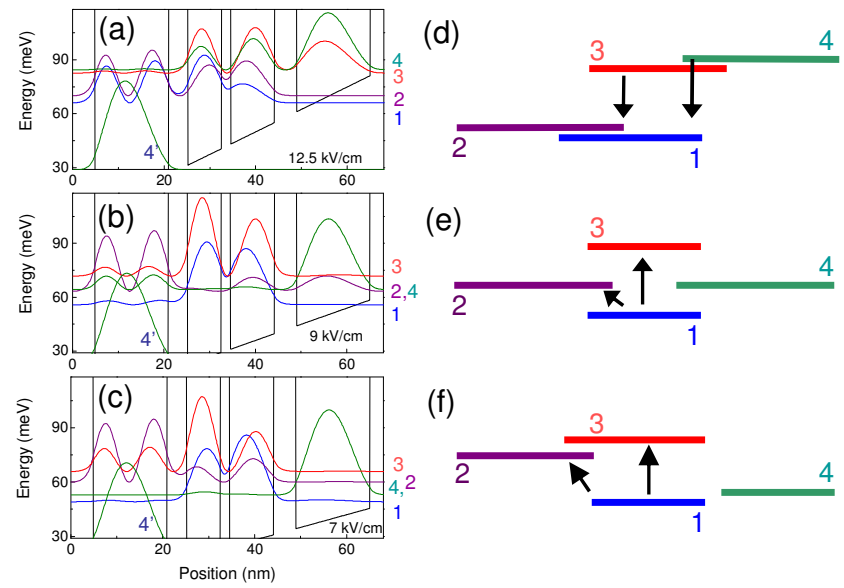


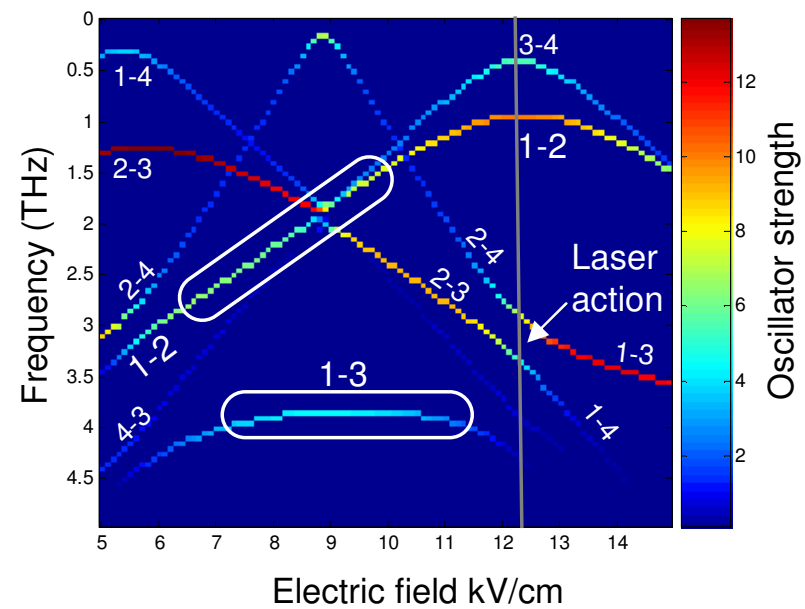

